Received 00th January 20xx, Accepted 00th January 20xx

DOI: $10.1039 / \times 0 \times x 00000 x$

\section{Single-molecule magnetism arising from cobalt(II) nodes of a crystalline sponge $\uparrow$}

\author{
Gabriel Brunet, Damir A. Safin, Jesús Jover, Eliseo Ruiz and Muralee Murugesu*
}

www.rsc.org/

The remarkable Metal-Organic Framework (MOF), $\left\{\left[\mathrm{Co}(\mathrm{NCS})_{2}\right)_{3}\left(\kappa^{3}-\right.\right.$ $\left.\left.\mathrm{TPT})_{4}\right] \cdot a\left(\mathrm{H}_{2} \mathrm{O}\right) \cdot b(\mathrm{MeOH})\right\}_{n}(1)$, which is used in the revolutionary crystalline sponge method, displays characteristic Single-Molecule Magnet (SMM) behaviour under applied static fields. We report the subtle effects of changes in the coordination environment of the Co" ions in 1, leading to drastically different magnetic behaviors of two additional related compounds, $\left\{\left[\mathrm{Co}(\mathrm{NCS})_{2}\right)_{3}\left(\mathrm{~K}^{0-3}\right.\right.$ TPT $\left.\left.)_{4}\right] \cdot c\left(\mathrm{H}_{2} \mathrm{O}\right)\right\}_{n} \quad$ (2) and $\left\{\left[\mathrm{Co}(\mathrm{NCS})_{2}\left(\mathrm{H}_{2} \mathrm{O}\right)_{0.65}(\mathrm{MeOH})_{0.35}\right)_{3}\left(\kappa^{3}-\right.\right.$ TPT $\left.\left.)_{2}\right] \cdot 2.4\left(\mathrm{H}_{2} \mathrm{O}\right)\right\}_{n}(3)$. Magnetic measurements reveal unquenched first order orbital angular momentum, leading to significant magnetic anisotropy in all compounds. Notably, the crystalline sponge is the first example of a 3D network built from Co" SingleIon Magnets (SIMs) as nodes.

In recent years, the drive towards molecular materials that behave as small nanomagnets has relied on the use of metal ions to generate non-zero spin ground states. The combination of large spin ground states with magnetic anisotropy can give rise to the magnet-like behaviour of slow relaxation of the magnetization. Molecular materials exhibiting such behaviour are commonly referred to as Single-Molecule Magnets (SMMs) or Single-lon Magnets (SIMS) for polynuclear and mononuclear complexes, respectively. ${ }^{1}$ When considering $3 d$ transition metal ions, magnetic anisotropy is commonly achieved by unquenched orbital angular momentum due to the unequal filling of the $d$ orbitals. ${ }^{2}$ In this regard, Co" ions in an octahedral ligand field are particularly interesting due to degenerate $t_{2 g}$ levels that are partially occupied, and thus orbital angular momentum is not quenched. An additional key parameter in the rational design of SMMs is control over the intermolecular interactions. Such interactions often hinder a precise understanding of the origin of the relaxation modes in SMMs, and moreover, can impede the observation of SMM-like behaviour. ${ }^{3}$ Consequently, several different approaches have been established

Department of Chemistry and Biomolecular Sciences, University of Ottawa, 10 Marie Curie, Ottawa, ON, Canada K1N 6N5, Email: m.murugesu@uottawa.ca; Tel: +1 (613) 5625800 ext. 2733

†Electronic Supplementary Information (ESI) available: Additional magnetic measurement plots are included. See DOI: 10.1039/x0xx00000x in order to minimize intermolecular interactions. Initially, the synthetic strategy consisted of incorporating a shell of peripheral protecting diamagnetic ligands and/or separating the spin carriers by large organic counterions. ${ }^{4}$ Another approach involves the magnetic dilution method which incorporates a paramagnetic ion into a diamagnetic system, effectively isolating a single paramagnetic metal center. ${ }^{5}$ A more recent strategy, however, involves fixing the metal centres in place through the use of rigid linkers that play the role of organic spacers. ${ }^{6}$ Subsequently, we can modulate the linkers to increase or decrease the space between spin carriers, leading to high dimensionality networks. Thus, MetalOrganic Frameworks (MOFs) provide a fascinating approach at potentially enhancing SMM properties. While MOFs are generally associated with applications based on gas storage and separation, ${ }^{7}$ the incorporation of magnetic moment carriers within the framework of a MOF, through either paramagnetic metal centres or radical linkers, can be an effective strategy towards fine-tuning the magnetic interactions between neighbouring moment carriers. ${ }^{8}$

Recently, a new subclass of MOFs, the so-called "crystalline sponges", were described in which guest encapsulation occurs in a single-crystal-to-single-crystal fashion, permitting the subsequent use of X-ray diffraction techniques to elucidate the crystal structure of the guest compound. ${ }^{9}$ Our investigations on the cobaltcontaining MOF $\left.\left\{\left[\mathrm{Co}(\mathrm{NCS})_{2}\right)_{3}\left(\kappa^{3}-\mathrm{TPT}\right)_{4}\right] \cdot a\left(\mathrm{H}_{2} \mathrm{O}\right) \cdot b(\mathrm{MeOH})\right\}_{n}(\mathbf{1})$, where TPT is 2,4,6-tris(4-pyridyl)-1,3,5-triazine, revealed two solid-stateto-solid-state transformations that significantly alter the structure and composition of the crystalline sponge. ${ }^{10}$ Nevertheless, we were intrigued by the potential of $\mathbf{1}$ to exhibit slow relaxation of the magnetization due to the octahedral ligand field of the Co" ions which promotes significant magnetic anisotropy as a result of unquenched first-order orbital angular momentum. Herein, we report the SMM behaviour of a crystalline sponge, which reveals the first example of a three-dimensional network built from Co" SIMs as nodes. The discovery of a crystalline sponge exhibiting SMM behaviour paves the way for novel guest encapsulation studies, where both dia- and paramagnetic guests can influence the 


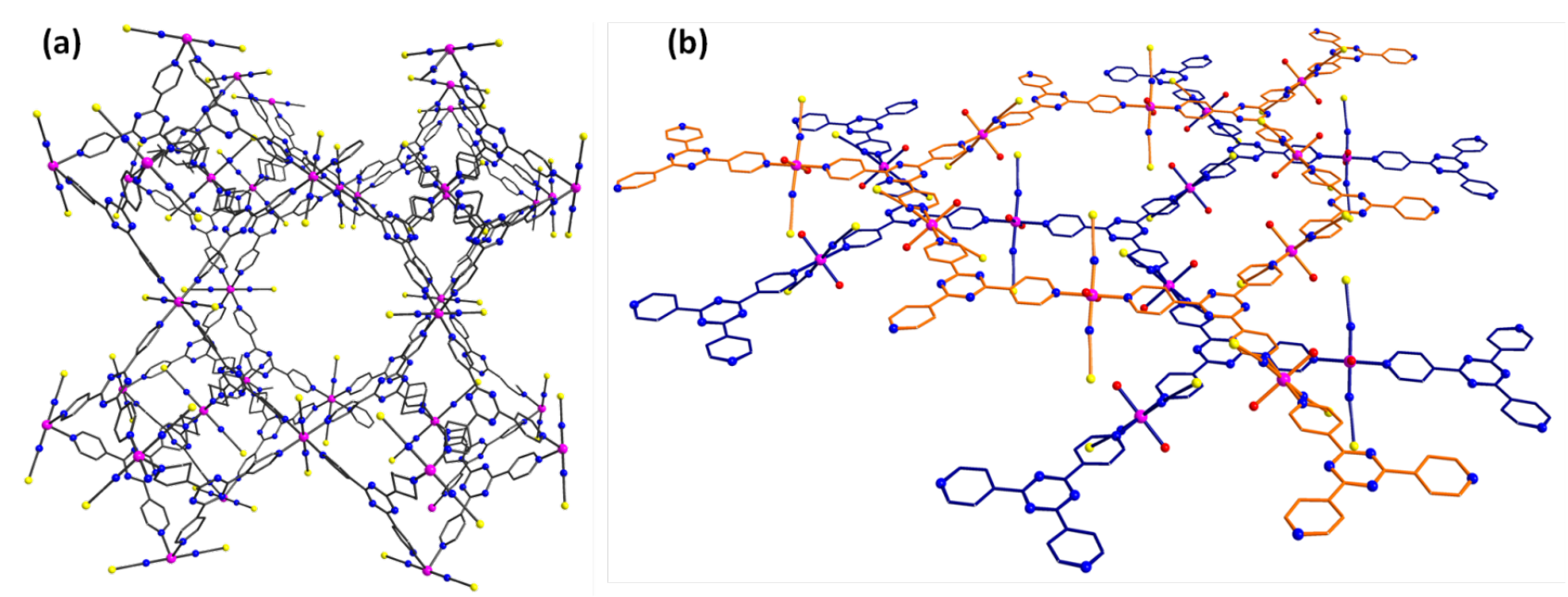

Fig. 1 (a) Packing arrangement of 1, illustrating the large pore dimensions of the 3D network. (b) View of the 2D planar sheet arrangement of 3, with individual sheets displayed in orange and blue. Colour code: purple (Co), blue (N), red (O), yellow (S). Carbon atoms are represented as stick model for clarity. Hydrogen atoms and solvent molecules are omitted for clarity.

from the parent MOF 1 through solid-state transformations, both of which exhibit frequency-dependent out-of-phase tails of signals, suggestive of SMM behaviour.

Compound 1 exhibits a 3D porous network, assembled by monomeric units of $\mathrm{CO}^{\prime \prime}$ in a slightly distorted octahedral coordination environment (Fig. 1). The TPT ligands take up the equatorial positions, while axial positions are occupied by nitrogen-bound thiocyanate anions. The Co" ions are wellisolated, with the closest Co $\cdots$ Co separation being $13.39 \AA$, which occurs through the TPT ligand (Fig. S1 in the ESI $\dagger$ ). Subsequently, we expect zero or minimal magnetic interactions between the metal centres. We have previously demonstrated that the removal of the single-crystals of $\mathbf{1}$ from solution, results in an irreversible transformation to a semiamorphous material in which the surface Co" ions undergo a change in coordination environment from octahedral to tetrahedral. ${ }^{10}$ This single-crystal-to-amorphous phase transition leads to the formation of $\left\{\left[\mathrm{Co}(\mathrm{NCS})_{2}\right)_{3}\left(\kappa^{0-3}\right.\right.$ $\left.\left.\mathrm{TPT})_{4}\right] \cdot c\left(\mathrm{H}_{2} \mathrm{O}\right)\right\}_{n}(2)$. The third and final compound studied in the present work, is obtained by evaporation of the $\mathrm{MeOH}$ layer during synthesis of $\mathbf{1}$, and yields the densely packed layered structure $\quad\left\{\left[\mathrm{Co}(\mathrm{NCS})_{2}\left(\mathrm{H}_{2} \mathrm{O}\right)_{0.65}(\mathrm{MeOH})_{0.35}\right)_{3}\left(\kappa^{3}\right.\right.$ TPT $\left.\left.)_{2}\right] \cdot 2.4\left(\mathrm{H}_{2} \mathrm{O}\right)\right\}_{n}(3)$ (Fig. 1). In this case, the Co" ions remain in a distorted octahedral symmetry, however, two TPT ligands have been replaced by coordinated water and methanol molecules. While the nearest intralayer Co $\cdots$ Co separation in $\mathbf{3}$ is $13.35 \AA$, the closest metal-metal distance is $8.37 \AA$ and occurs between adjacent sheets. A detailed description of the synthesis and structures of 1-3 can be found elsewhere. ${ }^{10}$

An analysis of the magnetic properties of $\mathbf{1}$ allows us to elucidate the effects of structural collapse due to solvent evaporation, as observed in $\mathbf{2}$, and of structural reorganization in $\mathbf{3}$, on the overall magnetic behaviour. It is important to note that magnetic measurements of $\mathbf{1}$ were performed in paraffin oil in order to prevent solvent evaporation and to maintain its structural integrity. Variable temperature direct current (dc) susceptibility measurements were performed at 1000 Oe in the temperature range of $1.8-300 \mathrm{~K}$ using a SQUID magnetometer (Fig. 2). The room temperature $\chi T$ products are 3.06, 2.64 and $2.94 \mathrm{~cm}^{3} \mathrm{~K} \mathrm{~mol}^{-1}$ for compounds $\mathbf{1}, 2$ and 3, respectively. These values, while higher than the anticipated spin-only value for $S=3 / 2$ of $1.88 \mathrm{~cm}^{3} \mathrm{~K} \mathrm{~mol}^{-1}$, still fall in an acceptable range when compared to other experimentally observed high-spin Co" ions with significant magnetic anisotropy. ${ }^{11}$ The $\chi T$ values remains fairly constant down to $200 \mathrm{~K}$ for all compounds investigated, before gradually decreasing upon further cooling. In all cases, the decrease of the $\chi^{T}$ product is most likely a consequence of magnetic anisotropy and/or thermal depopulation of the excited states

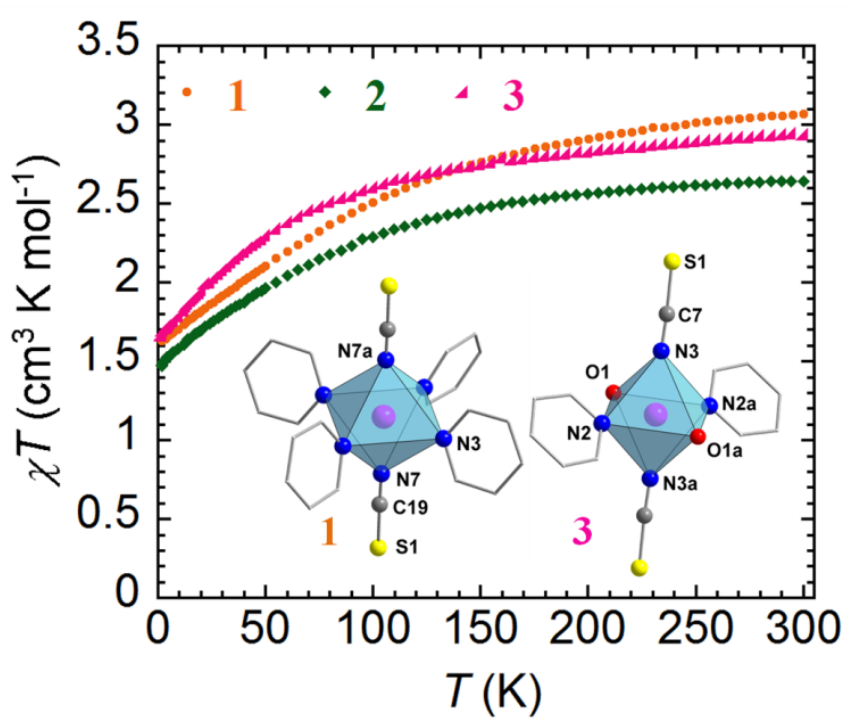


Fig. 2 Temperature dependence of the magnetic susceptibility for compounds 1-3 in a $\chi T$ vs. $T$ plot at 1000 Oe.

Table 1 Compilation of the energy barriers of recent octahedral Co" SIMs with extended structures (in one, two or three dimensions).

\begin{tabular}{|c|c|c|c|c|c|}
\hline Compound & Dimensionality & $\mathrm{SIM}, \mathrm{H} \neq 0$ (Oe) & $U_{\text {eff }}(\mathrm{K})$ & $\tau_{0}(\mathrm{~s})$ & Ref. \\
\hline$\left[\mathrm{Co}(\mathrm{btm})_{2}(\mathrm{SCN})_{2} \cdot \mathrm{H}_{2} \mathrm{O}\right]_{n}$ & $1 \mathrm{D}$ & 1500 & 45.4 & $5.6 \times 10^{-8}$ & $13 a$ \\
\hline$\left[\mathrm{Co}(\mathrm{azbbpy})(\mathrm{bpe})_{0.5}(\mathrm{DMF})(\mathrm{NCS})_{2}\right] \cdot 0.25 \mathrm{H}_{2} \mathrm{O}$ & $2 \mathrm{D}$ & 1000 & 8.4 & $1.7 \times 10^{-6}$ & $13 b$ \\
\hline$\left[\mathrm{Co}(\right.$ azbbpy $\left.)\left(4,4^{\prime} \text { bipy }\right)_{0.5}(\mathrm{DMF})(\mathrm{NCS})_{2}\right] \cdot \mathrm{MeOH}$ & $2 \mathrm{D}$ & 1000 & 14.0 & $1.2 \times 10^{-6}$ & $13 b$ \\
\hline$\left[\mathrm{Co}(\mathrm{dca})_{2}(\mathrm{atz})_{2}\right]_{n}$ & $2 \mathrm{D}$ & 1000 & 7.3 & $1.7 \times 10^{-6}$ & $13 c$ \\
\hline$\left[\mathrm{Co}(\mathrm{dca})_{2}(\mathrm{bim})_{2}\right]_{n}$ & $2 \mathrm{D}$ & $500-2500$ & $6.5-13.3$ & $0.37-1.54 \times 10^{-6}$ & $13 d$ \\
\hline$\left[\mathrm{Co}(\mathrm{dca})_{2}(\mathrm{bmim})_{2}\right]_{n}$ & $2 \mathrm{D}$ & $500-2500$ & $16.5-22.2$ & $6.3-7.2 \times 10^{-7}$ & $13 d$ \\
\hline$\left[\mathrm{Co}(\mathrm{ppad})_{2}\right]_{n}$ & $2 \mathrm{D}$ & 2000 & 16.4 & $5.0 \times 10^{-6}$ & $13 e$ \\
\hline$\left[\mathrm{Co}(\mathrm{pbeb})_{2}(\mathrm{NCS})_{2}\right] \cdot 7 \mathrm{DCB}$ & $2 \mathrm{D}$ & $250-1000$ & $\begin{array}{l}42.0-45.0 \\
10.9-12.7\end{array}$ & $\begin{array}{l}1.7-2.7 \times 10^{-8} \\
4.1-5.5 \times 10^{-6}\end{array}$ & $13 f$ \\
\hline$\left[\mathrm{Co}(\mathrm{pbeb})_{2}(\mathrm{NCS})_{2}\right] \cdot 4 \mathrm{TAN}$ & $2 \mathrm{D}$ & 1000 & $\begin{array}{c}24.6 \\
8.9\end{array}$ & $\begin{array}{l}2.3 \times 10^{-7} \\
7.8 \times 10^{-6}\end{array}$ & $13 f$ \\
\hline$\left[\mathrm{Co}(\mathrm{pbeb})_{2}(\mathrm{NCS})_{2}\right] \cdot 6 \mathrm{TOL}$ & $2 \mathrm{D}$ & 1000 & $\begin{array}{c}16.5 \\
3.6\end{array}$ & $\begin{array}{l}8.2 \times 10^{-7} \\
3.0 \times 10^{-5}\end{array}$ & $13 f$ \\
\hline$\left[\mathrm{Co}(\mathrm{pbeb})_{2}(\mathrm{NCS})_{2}\right] \cdot 8 \mathrm{PYR}$ & $2 \mathrm{D}$ & 1000 & $\begin{array}{c}30.2 \\
7.1\end{array}$ & $\begin{array}{l}1.3 \times 10^{-7} \\
1.0 \times 10^{-5}\end{array}$ & $13 f$ \\
\hline$\left.\left\{\left[\mathrm{Co}(\mathrm{NCS})_{2}\right)_{3}\left(\kappa^{3}-\mathrm{TPT}\right)_{4}\right] \cdot a\left(\mathrm{H}_{2} \mathrm{O}\right) \cdot b(\mathrm{MeOH})\right\}_{n}$ & $3 \mathrm{D}$ & 600 & 7.0 & $8.7 \times 10^{-6}$ & this work \\
\hline
\end{tabular}

rather than antiferromagnetic interactions due to the large distance separating the Co" ions. This is especially valid for 1 and 3, with the closest Co...Co distances being $13.39 \AA$ and $8.37 \AA$, respectively.

For 2, due to the structural rearrangement it is not possible to definitively rule out intermolecular interactions, however, based on the fact that $\mathbf{2}$ also contains tetrahedral Co" ions, non-negligible anisotropy can be expected. To confirm the presence of magnetic anisotropy, field dependent magnetization measurements $(M$ vs. $H)$ and reduced magnetization studies were performed on all compounds presented herein (Fig. S2-S4 in the ESI $\dagger$ ). In all cases, the magnetization curves reveal a rapid and steady increase of the magnetization at $1.8 \mathrm{~K}$ without clear saturation at $7 \mathrm{~T}$. The non-saturation, as well as the non-superimposition of the isotemperature lines in the $M v s . H / T$ data, clearly confirms the presence of significant magnetic anisotropy.

In recent years, mononuclear cobalt complexes with significant anisotropy were found to exhibit SMM-like behaviour. ${ }^{12,13}$ This behaviour is primarily arising from the inherent magnetic anisotropy of the metal centre which is strongly influenced by the ligand field and coordination geometry/environment. To investigate potential slow relaxation of the magnetization dynamics, temperature dependent alternating current (ac) susceptibilities were measured under applied fields of 0 and 1000 Oe for compounds 1-3 (Fig. S5-S7 in the ESI $\dagger$ ). For all compounds, an ac signal was only present under applied dc fields of 1000 Oe. This is generally indicative of the presence of significant quantum tunnelling of the magnetization due to non-negligible transverse anisotropy $(E)$. In the case of $\mathbf{1}$, the emergence of a clear peak, rather than merely tails of peaks, as in the case of $\mathbf{2}$ and 3, encouraged us to further examine the magnetic properties arising from this compound. The optimum applied dc field for 1, where the minimum of the characteristic frequency was observed, was determined to be $H_{\mathrm{dc}}=600 \mathrm{Oe}$ (Fig. S8 in the ESI $\dagger$ ). In the ac susceptibility data, the shifting of the peaks towards lower frequencies with decreasing temperatures is indicative of superparamagnet-like slow relaxation of a field-induced SMM (Fig. 3).

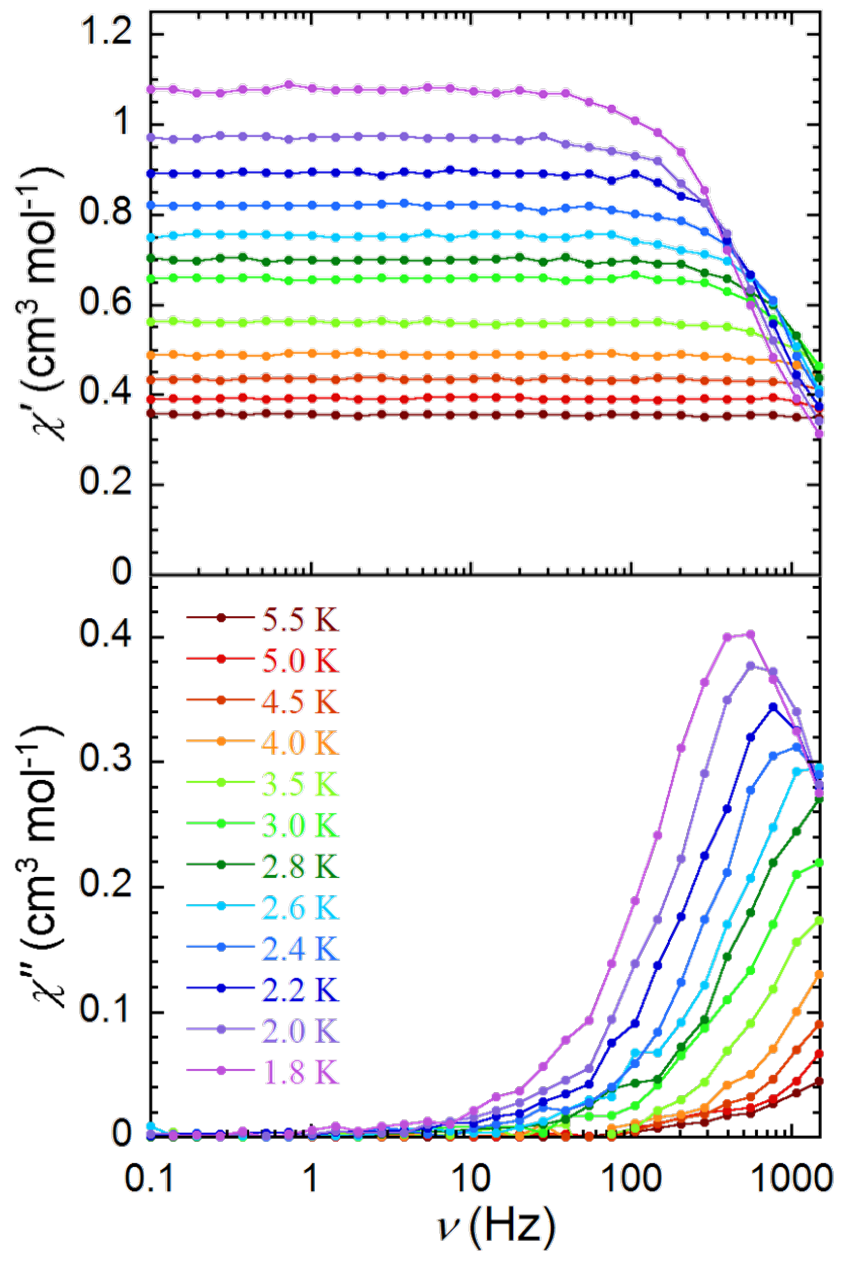

Fig. 3 Frequency dependence of the in-phase $\chi^{\prime}$ (top) and out-of-phase $\chi^{\prime \prime}$ (bottom) magnetic susceptibilities for 1 , under an applied optimum dc field of $H_{\mathrm{dc}}=600$ Oe. Lines serve as guides for the eyes. 
The thermally activated relaxation displays an Arrhenius-like behaviour $\left(\tau=\tau_{0} \exp \left(U_{\text {eff }} / k T\right)\right.$, where the anisotropy barrier obtained from the fitting is $U_{\text {eff }}=7.0 \mathrm{~K}\left(\tau_{0}=8.68 \times 10^{-6} \mathrm{~s}\right)$ (Fig. S9 in the ESI $\uparrow$ ). This observable barrier is rather small, yet comparable to other mononuclear Co" SMMs. ${ }^{2 b, 12}$ Such behaviour is in agreement with the predicted positive $D$ value for octahedral $d^{7}$ Co" cations, as demonstrated by Ruiz and coworkers. ${ }^{2 b}$ Recent energy barriers reported for Co"-based SIMs with structural dimensionalities greater than zero are summarized in Table 1 . To the best of our knowledge, 1 represents the first case of a 3D Co"-based network exhibiting SIM behaviour. The Cole-Cole plot $\left(\chi^{\prime \prime}\right.$ vs. $\left.\chi^{\prime}\right)$ of 1 was employed to confirm the presence of a single relaxation process (Fig. S10 in the ESI $\uparrow$ ). At fixed temperatures between 1.8 and $4 \mathrm{~K}$, semicircular plots were obtained and fitted using a generalized Debye model, yielding $\alpha$ parameters in the range of $0.01-0.13$, indicating a narrow distribution of relaxation times.

The disparity in the generation of a frequency dependent signal based on the application of an external applied field is often attributed to dipolar/hyperfine interactions and zerofield tunneling. In the case of $\mathbf{1}$, the large metal-metal separations would strongly suggest that the latter plays a significant part in suppressing SMM behavior at zero applied field. Nevertheless, slow magnetic relaxation can be revealed through the application of an external field. Thus, we were interested in the magnetic field dependence of the relaxation times. The Cole-Cole plot of the variable-field ac magnetic susceptibility data was fitted using a generalized Debye model (Fig. S11 in the ESI $\dagger$ ). The data could be fitted to give $\alpha \leq 0.085$ for the iso-field scans and a narrow distribution of relaxation times. This strongly suggests that the observed slow relaxation dynamics are dominated by a single process, which is not readily influenced by the application of a dc field, as evidenced by the consistent $\tau$ values at variable fields (Table S1 in the $\mathrm{ESI} \dagger$ ).

The observable difference in the generation of slow magnetic relaxation between $\mathbf{1}$ and $\mathbf{2}$ may be attributed to the change in coordination geometry from octahedral to tetrahedral. In theory, first-order orbital angular momentum, the principal contributor to magnetic anisotropy, is absent in a perfect tetrahedral geometry. However, it has been demonstrated that some distorted tetrahedral complexes exhibit non-negligible barriers even at zero applied dc fields due to the mixing of the electronic ground state and the anisotropic excited states. ${ }^{14}$ The sign of the anisotropy is often dictated by the ligand field around the metal centre. In our case, due to the amorphous nature of $\mathbf{2}$, it is not possible to identify any distortion in the coordination environment, and consequently, magneto-structural correlations cannot be performed. Nevertheless, through ac susceptibility measurements we can unequivocally conclude that $\mathbf{2}$ displays different structural features than the parent MOF 1 . When comparing the magnetic behaviours of $\mathbf{1}$ and $\mathbf{3}$, the weak ac signal observed for $\mathbf{3}$ can again be attributed to a change in the coordination environment of the Co" ions. In comparison to $\mathbf{1}$, two TPT nitrogen atoms are replaced by two oxygen atoms
Table 2. ORCA/CASSCF, ORCA/CASSCF + NEVPT2, and MOLCAS/CASSCF + RASSI computed $\mathrm{D},|\mathrm{E}|\left(\right.$ in $\left.\mathrm{cm}^{-1}\right)$, and g-values for the ground state of complexes $\mathbf{1}$ and $\mathbf{3}$. $\delta$ and $\Delta$ (in $\mathrm{cm}^{-1}$ ) are the computed first excitation energies before and after including the spin-orbit effects, respectively. The $\Delta$ value corresponds to the energy difference between the ground and the first excited Kramers' doublets.

\begin{tabular}{cccccc} 
Complex & $\mathrm{D}_{\text {calc }}$ & $|\mathrm{E}|_{\text {calc }}$ & $\delta$ & $\Delta$ & $\mathrm{g}_{\mathrm{xx}}, \mathrm{g}_{\mathrm{yy}}, \mathrm{g}_{\mathrm{zz}}$ \\
\hline $\mathbf{1}^{\mathrm{a}}$ & 148.1 & 5.6 & 157.3 & 296.9 & 1.592 .412 .82 \\
$\mathbf{1}^{\mathrm{b}}$ & 136.7 & 14.7 & 200.3 & 278.1 & 1.642 .352 .90 \\
$\mathbf{1}^{\mathrm{c}}$ & 136.6 & 29.2 & 165.9 & 291.2 & 1.762 .382 .88 \\
\hline $\mathbf{3}^{\mathrm{a}}$ & 99.8 & 18.2 & 379.2 & 209.3 & 1.802 .592 .98 \\
$\mathbf{3}^{\mathrm{b}}$ & 97.4 & 22.4 & 371.1 & 209.6 & 1.802 .502 .99 \\
$\mathbf{3}^{\mathrm{c}}$ & 99.1 & 24.4 & 354.4 & 215.4 & 1.802 .583 .02 \\
\hline
\end{tabular}

${ }^{a}$ ORCA/CASSCF. ${ }^{b}$ ORCA/CASSCF + NEVPT2. ${ }^{c}$ MOLCAS/CASSCF + RASSI.

from coordinated $\mathrm{H}_{2} \mathrm{O}$ and $\mathrm{MeOH}$ molecules. This change induces a weak ligand field around the metal centre and a smaller separation of the $t_{2 \mathrm{~g}}$ and $e_{\mathrm{g}}$ sets. Such a variation in the electronic configuration is known to lead to a change in the local anisotropy of the metal centre (i.e. sign and strength), which subsequently leads to weaker spin-orbital coupling. This results in a change of the superparamagnetic properties through a decrease of the energy barrier for magnetization reversal.

The magnetic properties of the low-lying states of complexes 1 and 3 were analyzed by means of an $a b$ initio multireference methodology; the computed second-order anisotropy parameters and excitation energies are collected in Table 2. These values have been obtained from two different electronic structure calculations that have been carried out with the ORCA $^{15}$ and MOLCAS ${ }^{16}$ software packages. ORCA produces two sets of results: CASSCF and CASSCF + NEVPT2 (which introduces the dynamic correlation effects), both including spin-orbit contributions. On the other hand MOLCAS has been only able to provide CASSCF results, including spin-orbit effects that have been introduced with the SO-RASSI method. As expected for octahedral Co" complexes large and positive D values are found. ${ }^{2 b}$ The computed $D$ parameters remain very similar regardless the method employed and are larger for complex 1 . These computed values are not unusual since the spin relaxation mechanisms that depend on the lattice effects, and should contribute to reduce the $D$ values, cannot be captured in a single-molecule calculation. In all cases, a 3/2 ground state is found for both complexes before including the spin-orbit effects. In these conditions, the calculations show the existence of low-lying spin-orbit free excited states ( $\delta$ in Table 2) with close energies to the ground state, which may be responsible of the observed anisotropy. This is also confirmed by the anisotropic g-values for the ground state of $\mathbf{1}$ and $\mathbf{3}$ (Table 2). Once the spin-orbit effects are included a set of Kramers' doublets (KDs, D) for each complex is obtained. In the case of complex 1 there are two low-lying KDs at around 280 and $450 \mathrm{~cm}^{-1}$, which may participate in the spin relaxation processes (see below). This situation changes slightly for complex 3; while the first KD lies low at around $210 \mathrm{~cm}^{-1}$ the second excited state quite higher in energy $\left(650 \mathrm{~cm}^{-1}\right)$ and is probably not able to intervene in the relaxation mechanism. A complete list of g-values, D-contributions, $\delta$ and $\Delta$ for 


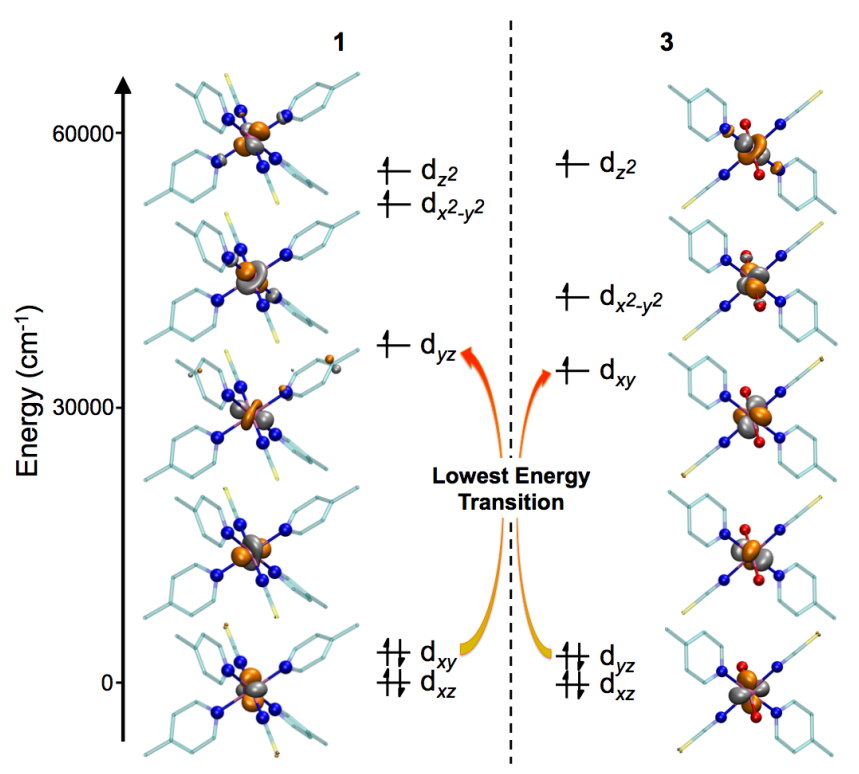

Fig. 4 Co" core and computed d-orbital splitting for complexes 1 (left) and 3 (right). Color code: $\mathrm{Co}=$ pink $\mathrm{C}=$ light blue, $\mathrm{N}=$ blue, $\mathrm{O}=$ red, $\mathrm{S}=$ yellow. $; \mathrm{H}$ atoms have been omitted for clarity.

complexes $\mathbf{1}$ and 3, as well as the orientation of the g- and Dtensors, can be found in the Supporting Information file (Tables S2-7 and Figure S12).

Extracting the excitation energies from CASSCF calculations is relatively easy and fast; nevertheless, identifying the metal dorbitals involved in such transitions is not straightforward. For that reason using a single-determinant wavefunction calculation (DFT) is often the method of choice for obtaining a qualitative explanation of the excitation processes, in which the orbital composition is much easier to rationalize. By doing this, the excitation energies correspond to electronic transitions from the highest energy doubly occupied orbital to the higher energy semioccupied $\beta$-orbitals. The DFT calculations of complexes $\mathbf{1}$ and $\mathbf{3}$ have been carried out with the g09 package. ${ }^{17}$ The final d-orbital splitting of the studied complexes, which allows the location of the lowest energy transitions, is shown in Figure 4. As may be observed, the degeneracy of the $t_{2 g}$ orbitals is broken and one of those moves up in energy, far from the last doubly occupied orbitals. In the case of complex $\mathbf{1}$ the last doubly occupied orbital is $d_{x y}$ and the first semioccupied orbital is $d_{y z}$ (or $d_{x z}$, because those cannot be distinguished). Since these orbitals have a different $\left|m_{1}\right|$ value i.e. \pm 2 and \pm 1 , respectively, the $D$ value should be positive. The reverse situation is found in complex $\mathbf{3}$, in this case the highest energy doubly occupied orbital is $d_{y z}$ (or $d_{x z}$ ) while the lowest energy semioccupied orbital is $d_{x y}$. As before a transition between these orbital entails a change in $\left|m_{1}\right|$, thus producing a positive $D$ value. These computed d-orbital splitting schemes confirm that the ligand field i.e. the separation between $t_{2 g}$ and $e_{g}$ orbitals is smaller in complex $\mathbf{3}$, in agreement with the experimental observations.

The computed relative energies of the lowest-lying KDs and the spin relaxation pathways of $\mathbf{1}$ and $\mathbf{3}$ are shown in Figure 5 .
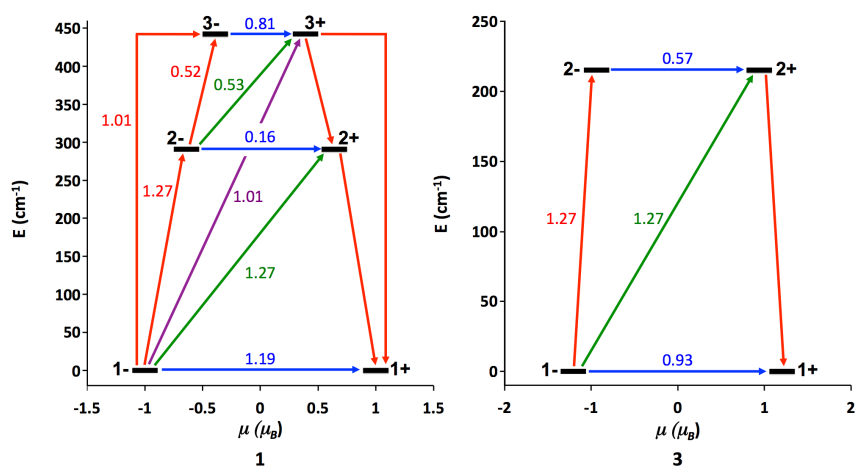

Fig. 5 Lowest Kramers' doublets and ab initio computed relaxation mechanism in 1 (left) and $\mathbf{3}$ (right). The thick black lines imply KDs as a function of their magnetic moment along the main anisotropy axis. Red lines indicate the magnetization reversal mechanism. The blue lines correspond to ground state QTM and thermally assistedQTM via the first and second excited KD, and green and purple lines show possible Orbach relaxation processes. The values close to the arrows indicate the matrix elements of the transition magnetic moments (above 0.1 an efficient spin relaxation mechanism is expected). ${ }^{18}$

In both cases the spin relaxation mechanisms show a plausible pathway via a direct quantum tunneling (QTM) in the ground state, as proposed from experiments. The matrix elements of the transition magnetic moments between states 1 - and $1+$ are 1.19 and 0.93 for $\mathbf{1}$ and $\mathbf{3}$, respectively, much higher that the 0.1 required value associated to an efficient relaxation mechanism. In the case of $\mathbf{1}$, the first two KDs may be accessible (aprox. 280 and $450 \mathrm{~cm}^{-1}$ ) and able to participate in alternative relaxation pathways, either thermally assisted-QTM or Orbach processes. In complex $\mathbf{3}$ there is only one low-lying $K D$ at around $210 \mathrm{~cm}^{-1}$ (the second lowest KD is located at almost $650 \mathrm{~cm}^{-1}$ ); the alternative thermally assisted-QTM and Orbach spin relaxation processes seem plausible but are probably not able to compete with the ground state QTM. These relaxation processes provide an explanation to the relatively low experimentally observed $U_{\text {eff }}$ values despite the relatively large D values of the Co" complexes studied.

\section{Conclusions}

We have reported the magnetic properties of three Co" compounds, which were fully characterized through dc, ac susceptibility measurements abd electronic structure calculations. Interestingly, a 3D crystalline sponge displays single-molecule magnet-like behaviour under applied static field, where each node individually acts as a nanomagnet. We have also demonstrated that the magnet-like behaviour of these nodes can be fine-tuned via manipulation of the coordination environment of the $\mathrm{Co}^{\prime \prime}$ ions. Thus, changes in the coordination sphere of metal centres in extended networks could be easily monitored through their magnetic properties. Furthermore, we can envision how the magnetization dynamics of the porous host could be tuned by guest exchange and similarly, how the intercalation of guest molecules could be detected via magnetism for novel sensor-based application. 


\section{Acknowledgements}

We wish to acknowledge the financial support from NSERC, $\mathrm{CFI}$, the University of Ottawa and the Spanish Ministerio de Economía y Competitividad (grant CTQ2015-64579-C3-1-P, MINECO/FEDER, UE). E.R. thanks Generalitat de Catalunya for an ICREA Academia award. J.J and E.R. thankfully acknowledge the computer resources in the Consorci Serveis Universitaris de Catalunya (CSUC).

\section{References}

1 D. Gatteschi, R. Sessoli and J. Villain, Molecular Nanomagnets, OUP, 2006.

2 (a) G. A. Craig and M. Murrie, Chem. Soc. Rev., 2015, 44, 2135; (b) S. Gomez-Coca, E. Cremades, N. Aliaga-Alcade and E. Ruiz, J. Am. Chem. Soc., 2013, 135, 7010; (c) M. Atanasov, D. Aravena, E. Suturina, E. Bill, D. Maganas and F. Neese, Coord. Chem. Rev., 2015, 289-290, 177.

3 (a) K. E. Vostrikova and D. Luneau, J. Am. Chem. Soc., 2000, 122, 718; (b) A. M. Ako, V. Mereacre, I. J. Hewitt, R. Clérac, L. Lecren, C. E. Anson and A. K. Powell, J. Mater. Chem., 2006, 16, 2579; (c) D. Luneau and P. Rey, Coord. Chem. Rev., 2005, 249, 2591.

4 (a) T. Jurca, A. Farghal, P.-H. Lin, I. Korobkov, M. Murugesu and D. S. Richeson, J. Am. Chem. Soc., 2011, 133, 15814; (b) G. Christou, Polyhedron, 2005, 24, 2065; (c) C. R. Ganivet, B. Ballesteros, G. de la Torre, J. M. Clemente-Juan, E. Coronado and T. Torres, Chem. Eur. J., 2013, 19, 1457.

5 (a) F. Habib, P.-H. Lin, J. Long, I. Korobkov, W. Wernsdorfer and M. Murugesu, J. Am. Chem. Soc., 2011, 133, 8830; (b) S. D. Jiang, B.-W. Wang, G. Su, Z.-M. Wang and S. Gao, Angew. Chem., 2010, 122, 7610; . (c) K. R. Meihaus, J. D. Rinehart and J. R. Long, Inorg. Chem., 2011, 50, 8484.

6 (a) M. Kurmoo, Chem. Soc. Rev., 2009, 39, 1353; (b) M.-H. Zeng, B. Wang, X.-Y. Wang, W.-X. Zhang, X.-M. Chen and S. Gao, Inorg. Chem., 2006, 45, 7069; (c) M. Eddaoudi, D. B. Moler, H. Li, B. Chen, T. M. Reineke, M. O'Keeffe and O. M. Yaghi, Acc. Chem. Res., 2001, 34, 319.

7 (a) J.-R. Li, R. J. Kuppler and H.-C. Zhou, Chem. Soc. Rev., 2009, 38, 1477; (b) U. Mueller, M. Schubert, F. Teich, H. Puetter, K. Schierle-Arndt and J. Pastré, J. Mater. Chem., 2006, 16, 626; (c) H. Furukawa, K. E. Cordova, M. O'Keeffe and O. M. Yaghi, Science, 2013, 341, 1230444

8 (a) X. Zhang, V. Vieru, X. Feng, J.-L. Liu, Z. Zhang, B. Na, W. Shi, B.-W. Wang, A. K. Powell, L. F. Chibotaru, S. Gao, P. Cheng and J. R. Long, Angew. Chem. Int. Ed., 2015, 54, 9861; (b) R. Sibille, T. Mazet, B. Malaman, T. Gaudisson and M. François, Inorg. Chem., 2012, 51, 2885; (c) Z.-L. Huang, M. Drillon, N. Masciocchi, A. Sironi, J.-T. Zhao, P. Rabu and P. Panissod, Chem. Mater., 2000, 12, 2805.

9 Y. Inokuma, S. Yoshioka, J. Ariyoshi, T. Arai, Y. Hitora, K. Takada, S. Matsuanaga, K. Rissanen and M. Fujita, Nature, 2013, 495, 461.

10 G. Brunet, D. A. Safin, I. Korobkov, A. Cognigni and M. Murugesu, Cryst. Growth Des., 2016, DOI: 10.1021/acs.cgd.6b00570.
11 (a) T.-F. Liu, D. Fu, S. Gao, Y.-Z. Zhang, H. L. Sun, G. Su and Y.J. Liu, J. Am. Chem. Soc., 2003, 125, 13976; (b) F. Habib, C. Cook, I. Korobkov and M. Murugesu, Inorg. Chim. Acta., 2012, 380, 378; (c) J. Vallejo, I. Castro, R. Ruiz-García, J. Cano, M. Julve, F. Lloret, G. De Munno, W. Wernsdorfer and E. Pardo, J. Am. Chem. Soc., 2012, 134, 15704; (d) R. Herchel, L. Váhovská, I. Potočňák and Z. Trávníček, Inorg. Chem., 2014, 53, 5896.

12 J. M. Zadrozny, J. Liu, N. A. Piro, C. J. Chang, S. Hill, J. R. Long, Chem. Commun., 2012, 48, 3927.

13 (a) Y.-Y. Zhu, M.-S. Zhu, T.-T. Yin, Y.-S. Meng, Z.-Q. Wu, Y.-Q. Zhang and S. Gao, Inorg. Chem., 2015, 54, 3716; (b) A. E. Ion, S. Nica, A. M. Madalan, S. Shova, J. Vallejo, M. Julve, F. Lloret and M. Andruh, Inorg. Chem., 2015, 54, 16; (c) J. PalionGazda, T. Klemens, B. Machura, J. Vallejo, F. Lloret and M. Julve, Dalton Trans., 2015, 44, 2989; (d) A. ŚwitlickaOlszewska, J. Palion-Gazda, T. Klemens, B. Machura, J. Vallejo, J. Cano, F. Lloret and M. Julve, Dalton Trans., 2016, 45, 10181; (e) X. Liu, L. Sun, H. Zhou, P. Cen, X. Jin, G. Xie, S. Chen and Q. Hu, Inorg. Chem., 2015, 54, 8884; (f) J. Vallejo, F. R. Fortea-Pérez, E. Pardo, S. Benmansour, I. Castro, J. Krzystek, D. Armentano and J. Cano, Chem. Sci., 2016, 7, 2286.

14 (a) M. R. Saber and K. R. Dunbar, Chem. Commun., 2014, 50, 12266; (b) S. Vaidya, A. Upadhyay, S. K. Singh, T. Gupta, S. Tewary, S. K. Langley, J. P. S. Walsh, K. S. Murray, G. Rajaraman and M. Shanmugam, Chem. Commun., 2015, 51, 3739.

15 F. Neese, WIREs Comput. Mol. Sci., 2012, 2, 73.

16 (a) G. Karlström, R. Lindh, P.-A.. Malmqvist, B. O. Roos, U. Ryde, V. Veryazov, P.-O. Widmark, M. Cossi, B. Schimmelpfennig, P. Neogrady and L. Seijo, Comput. Matter Sci., 2003, 28, 222; (b) V. Veryazov, P.-O. Widmark, L. Serrano-Andrés, R. Lindh and B. O. Roos, Int. J. Quantum Chem., 2004, 100, 626; (c) F. Aquilante, L. De Vico, N. Ferré, G. Ghigo, P.-å. Malmqvist, P. Neogrády, T. B. Pedersen, M. Pitoňák, M. Reiher, B. O. Roos, L. Serrano-Andrés, M. Urban, V. Veryazov and R. Lindh, J. Comp. Chem., 2010, 31, 224.

17 M. J. Frisch, G. W. Trucks, H. B. Schlegel, G. E. Scuseria, M. A. Robb, J. R. Cheeseman, G. Scalmani, V. Barone, B. Mennucci, G. A. Petersson, H. Nakatsuji, M. Caricato, X. Li, H. P. Hratchian, A. F. Izmaylov, J. Bloino, G. Zheng, J. L. Sonnenberg, M. Hada, M. Ehara, K. Toyota, R. Fukuda, J. Hasegawa, M. Ishida, T. Nakajima, Y. Honda, O. Kitao, H. Nakai, T. Vreven, J. Montgomery, J. A., J. E. Peralta, F. Ogliaro, M. Bearpark, J. J. Heyd, E. Brothers, K. N. Kudin, V. N. Staroverov, R. Kobayashi, J. Normand, K. Raghavachari, A. Rendell, J. C. Burant, S. S. Iyengar, J. Tomasi, M. Cossi, N. Rega, N. J. Millam, M. Klene, J. E. Knox, J. B. Cross, V. Bakken, C. Adamo, J. Jaramillo, R. Gomperts, R. E. Stratmann, O. Yazyev, A. J. Austin, R. Cammi, C. Pomelli, J. W. Ochterski, R. L. Martin, K. Morokuma, V. G. Zakrzewski, G. A. Voth, P. Salvador, J. J. Dannenberg, S. Dapprich, A. D. Daniels, Ö. Farkas, J. B. Foresman, J. V. Ortiz and J. F. Cioslowski, D. J. Gaussian09, Revision D.01, Gaussian, Inc.: Wallingford CT, 2009.

18 S. Gómez-Coca, D. Aravena, R. Morales and E. Ruiz, Coord. Chem. Rev., 2015, 289-290, 379. 